\title{
Altruism, Dispersal, and Phenotype-Matching Kin Recognition
}

\author{
Laurent Lehmann and Nicolas Perrin ${ }^{\star}$
}

Institute of Ecology, University of Lausanne, CH-1015 Lausanne, Switzerland

Submitted March 20, 2001; Accepted November 13, 2001

ABSTRACT: We investigate the coevolution between philopatry and altruism in island-model populations when kin recognition occurs through phenotype matching. In saturated environments, a good discrimination ability is a necessary prerequisite for the emergence of sociality. Discrimination decreases not only with the average phenotypic similarity between immigrants and residents (i.e., with environmental homogeneity and past gene flow) but also with the sampling variance of similarity distributions (a negative function of the number of traits sampled). Whether discrimination should rely on genetically or environmentally determined traits depends on the apportionment of phenotypic variance and, in particular, on the relative values of $e$ (the among-group component of environmental variance) and $r$ (the among-group component of genetic variance, which also measures relatedness among group members). If $r$ exceeds $e$, highly heritable cues do better. Discrimination and altruism, however, remain low unless philopatry is enforced by ecological constraints. If $e$ exceeds $r$, by contrast, nonheritable traits do better. High $e$ values improve discrimination drastically and thus have the potential to drive sociality, even in the absence of ecological constraints. The emergence of sociality thus can be facilitated by enhancing $e$, which we argue is the main purpose of cue standardization within groups, as observed in many social insects, birds, and mammals, including humans.

Keywords: game theory, heritability, inclusive fitness, kin competition, quantitative genetics, social evolution.

Altruistic interactions, in which actors sacrifice some of their individual fitness to the benefit of recipients, cannot evolve unless interacting individuals are more related than average in the population (Hamilton 1964). Moreover, in a saturated environment, the population viscosity induced by low dispersal is unable to create conditions conducive to altruism. Though neighbors are indeed more likely to be relatives, they are also more likely to be competitors so

\footnotetext{
* E-mail: Nicolas.Perrin@ie-zea.unil.ch.
}

Am. Nat. 2002. Vol. 159, pp. 451-468. (c) 2002 by The University of Chicago. 0003-0147/2002/15905-0003\$15.00. All rights reserved.

that the two effects exactly cancel out (Taylor 1992; Wilson et al. 1992). For altruism to evolve, social and economic neighborhoods must be disentangled; that is, relatedness among partners involved in altruistic interactions must exceed that among partners involved in competitive interactions (Queller 1992, 1994).

This can be achieved if a kin-discrimination mechanism allows individuals to choose social partners according to kinship-related cues, while competitive interactions remain random. Perrin and Lehmann (2001) investigate the coevolution of altruism and dispersal under two contrasting kin-discrimination abilities. A spatially based discrimination, in which actors behave altruistically to anyone met within their natal patch (but not outside), does allow some altruism to evolve but at a low level and only insofar as ecological constraints (dispersal costs) prevent kin competition to drive complete dispersal. By contrast, associative learning, by which actors learn to recognize individually all conspecifics born on the same natal patch, promotes high levels of philopatry and altruism, even in the absence of dispersal costs; as discrimination improves, altruism provides enough inclusive benefits to overcome the costs of kin competition and thereby enforce philopatry.

Natural selection should in principle select the best discrimination abilities, but their evolution might be constrained. Perfect associative learning requires a set of restrictive conditions (including extended cognitive abilities, long developmental period, and small group size), which may be fulfilled in higher-vertebrate societies (those of birds and mammals) but certainly not in most invertebrates, in which individual recognition is usually not feasible. Instead, discrimination may often rely on kinshipindicative traits, mediated, for example, by visual, auditive, or olfactive cues.

\section{Genetic versus Environmental Cues}

Genetically based kin discrimination has been found in several groups of marine clonal invertebrates (references in Crozier 1986 and Grosberg 1988). Larvae of the sessile colonial ascidian Botryllus schlosseri distinguish kin on the basis of shared alleles at a highly polymorphic histocom- 
patibility locus (Grosberg and Quinn 1986). Mice have also been shown to rely on histocompatibility genes (MHC) for inbreeding avoidance (Potts et al. 1991) as well as for cooperation (Manning et al. 1992). Cannibalistic larvae of the tiger salamander Ambystoma tigrinum use genetically based sibship-specific olfactory signals to feed voraciously on nonrelatives but avoid eating close kin (Pfennig et al. 1994). Genetic components of recognition have also been found in arthropods, including subsocial isopods (Lisenmair 1987); cannibalizing coccinellids (Joseph et al. 1999); and eusocial bees (e.g., Greenberg 1979; Moritz and Hillesheim 1990), ants (e.g., Beye et al. 1998; Tsutsui et al. 2000), and termites (e.g., Adams 1991).

Similarities among nest mates, however, may also stem from common environment (as opposed to common ancestry) since individuals reared in the same colony normally share both. Such environmental components of colony recognition have been evidenced in many social species, including ants (e.g., Jutsum et al. 1979; Obin 1986; Crosland 1989a), wasps (e.g., Gamboa et al. 1986, 1996), and bees (e.g., Breed et al. 1995, plus further references in Ratnieks 1991). Eusocial naked mole rats also use environmental cues to discriminate colony members from those of neighboring colonies (O'riain and Jarvis 1997). In humans, discrimination may occur on the basis of cultural traits (e.g., local dialects, behavioral habits, or traditional ornaments), which are purely environmental.

\section{Phenotype Matching and Acceptance Threshold}

The mechanism underlying group recognition has been described as "phenotype matching" (Lacy and Sherman 1983); individuals compare the phenotype of potential partners with an inner template, checking for similarities or dissimilarities. This mechanism (and, in particular, selfreferent phenotype matching, the so-called armpit effect) has received strong empirical support (e.g., Heth et al. 1998; Hauber and Sherman 2000; Mateo and Johnston 2000). Phenotype matching is obviously not error proof (Lacy and Sherman 1983; Reeve 1989). The similarity distributions of kin and nonkin with the focal individual's template may overlap. An unrelated immigrant met on the natal patch might turn out to be, by chance, more similar to the focal individual than a related resident. Two types of errors are therefore possible: that of accepting an undesirable partner and that of rejecting a desirable one. Natural selection should favor the evolution of an optimal acceptance threshold (similarity level above which potential partners are accepted) that minimizes the two types of errors. However, depending on the costs and benefits of altruism, these errors presumably have not the same importance and should be weighed differentially.

Investigating evolutionarily stable strategies (ESS) for acceptance thresholds under a variety of scenarios ("recognition contexts"), Reeve (1989) showed these to depend on the relatedness of actor to potential recipients, the relative frequency of interactions with differently related classes of recipients, and the fitness consequences of accepting or rejecting a potential recipient. Namely, acceptance thresholds should decrease (i.e., actors be more tolerant) with increasing relatedness to desirable recipients, frequency of interactions with them (relative to those with nondesirable partners), and benefits of altruism.

\section{Complex Interactions}

Relatedness, interaction frequencies, altruism investments, and similarity distributions were fixed quantities in Reeve's (1989) model. However, as argued below, all four would be more realistically considered as interacting variables and are expected to coevolve with acceptance thresholds.

First, the optimal level of altruism conceded, and thereby both the costs and benefits involved in altruistic interactions should depend on the frequency of errors when conceding altruism (a function of both similarity distributions and threshold value), in addition to their dependence on relatedness and interaction frequencies.

Second, the frequency of interactions with kin versus nonkin (or less kin) is determined by population viscosity and dispersal patterns, which, in turn, depend on relatedness and altruism. While the kin-competition pressure induced by local relatedness enforces dispersal (Hamilton and May 1977), the benefits of altruistic interactions with kin may restrain it (the "benefits-of-philopatry"; Stacey and Ligon 1991).

Third, relatedness itself is a dynamic variable, depending on dispersal patterns, benefits of altruism, and error rate. Indeed, residents are always more likely than immigrants to interact with relatives. High benefits as well as low error rates thus increase the fitness of residents (their relative contribution to local gene pool), which boosts local relatedness.

Last, similarity distributions are expected to depend on local relatedness, insofar as recognition traits have a genetic component. The higher the dispersal rates, the lower the genetic component of similarity among patch mates, and the more overlap between similarity distributions of immigrants and residents. This should presumably refrain the evolution of altruism and thereby further boost dispersal.

In this article, we formalize the coevolutionary patterns between altruism, dispersal, and acceptance threshold, while accounting for the dynamic component of relatedness and similarity distributions. This allows us to evaluate the role of the number of recognition traits and their architecture (i.e., their heritability, as well as the part of 
similarity among patch mates due to common environment) in the establishment of social interactions. We investigate, in particular, whether and how much phenotypic overlap in indicative traits among residents and immigrants may impede the evolution of altruism, and we estimate how dispersal costs and local group sizes may affect the outcome of evolutionary paths.

\section{The Model}

\section{Model Organism}

We consider diploid individuals living in patches of limited size (say, $N$ breeding spots per patch), which induces local kin structures. Patches are numerous enough that coancestry among them can be neglected (infinite-island population model). The life cycle is annual, with events occurring in the following order:

Offspring spend a juvenile period in their place of birth, where they fully develop phenotypic features. Similarity among patch mates arises from common genes and/or environment.

At maturity, they disperse before reproduction with probability $x$ (that may evolve) and survive dispersal with probability $s$. Individuals disperse at random with respect to which patch is reached, then mate at random on that patch. Males take no further role in reproduction.

Resident and immigrant females then compete on each patch for the $N$ breeding spots.

Social interactions occur pairwise among successful females. Individuals meet a potential recipient, and based on phenotype matching (see "Phenotype Matching"), either accept it (i.e., provide altruism) or reject it (provide nothing).

Fecundity is affected by the outcome of altruism. The baseline fecundity of the focal female is devaluated by an amount $C$ if she provided help and incremented by an amount $B$ if she benefited from the help of a patch mate. (Symbols are defined in table A1.)

\section{Phenotype Matching}

Phenotype matching relies on the similarity between the focal individual and potential recipients with respect to a series (say, $n$ ) of uncorrelated recognition traits. This section is aimed at generating the distribution of similarities for immigrants and residents. Traits are assumed to be normally distributed and comprise both environmental and genetic components (the latter are assumed to be additive only, for simplicity):

$$
z_{\bullet}=a_{\bullet}+e_{\bullet},
$$

where $z_{\bullet}$ is the phenotypic value of a trait in a focal individual born on patch $j, a_{\bullet}$ is its genetic component (breeding value), and $e_{\bullet}$ is its environmental component. To simplify notations (and without loss of generality), phenotypic values are standardized over the whole population (i.e., they have grand mean 0 and variance 1 ). Different patches present different distributions because both the genetic and the environmental components have a between-group and a within-group part:

$$
\begin{aligned}
& a_{\bullet}=a_{j}+a_{\circ}, \\
& e_{\bullet}=e_{j}+e_{\circ},
\end{aligned}
$$

where $a_{j}$ is the average breeding value in patch $j$ and $a_{\circ}$ is the deviation of the focal individual's breeding value from its patch average. Similarly, $e_{i}$ is the average environmental value in patch $j$, and $e_{\circ}$ is the individual environmental deviation from it.

From the point of view of a focal individual, the maximum likelihood estimate of its similarity with a patch mate $y$ is given by the coefficient of regression $\eta$ (Lynch and Walsh 1998, app. 4) of the phenotype of $y$ on its own phenotype:

$$
\eta=\frac{\sum\left(a_{j}+a_{y}+e_{j}+e_{y}\right)\left(a_{j}+a_{\circ}+e_{j}+e_{\circ}\right)}{\sum\left(a_{j}+a_{\circ}+e_{j}+e_{\circ}\right)^{2}},
$$

where $a_{y}$ and $\mathrm{e}_{y}$ are the deviations of individual $y$ from patch-average breeding and environmental values, respectively, and sums are taken over the $n$ recognition traits. As traits are standardized and uncorrelated, the expectation of $\eta$ is given by

$$
\bar{\eta}=\frac{\sigma_{a_{\mathrm{b}}}^{2}+\sigma_{e_{\mathrm{b}}}^{2}}{\sigma_{z}^{2}},
$$

where the expected variance within individuals over traits $\left(\sigma_{z}^{2}\right)$ equals 1 . This variance splits up into several components: $\sigma_{z}^{2}=\sigma_{a}^{2}+\sigma_{e}^{2}$, where $\sigma_{a}^{2}=\sigma_{a_{\mathrm{b}}}^{2}+\sigma_{a_{\mathrm{v}}}^{2}$ is the total genetic variance and $\sigma_{e}^{2}=\sigma_{e_{\mathrm{b}}}^{2}+\sigma_{e_{\mathrm{w}}}^{2}$ is the total environmental variance. The terms $\sigma_{a_{\mathrm{b}}}^{2}$ and $\sigma_{a_{\mathrm{v}}}^{2}$ represent the between- and within-group components of genetic variance, respectively, while $\sigma_{e_{b}}^{2}$ and $\sigma_{e_{w}}^{2}$ represent the betweenand within-group components of environmental variance. No covariances appear in (2b) because genetic and environmental components are assumed independent, and individual deviations are residual variables. Equation (2b) may be written as

$$
\bar{\eta}=r h^{2}+e\left(1-h^{2}\right)
$$

where $r=\operatorname{cov}_{a} / \sigma_{a}^{2}=\sigma_{a_{b}}^{2} / \sigma_{a}^{2}$ measures the relatedness of 
the focal individual to its patch mates, obtained as the regression of breeding values (equal to the proportion of genetic variance due to among-patches differences), $h^{2}=\sigma_{a}^{2} / \sigma_{z}^{2}$ is the heritability of the traits (proportion of phenotypic variance that is additive genetic), $e=$ $\operatorname{cov}_{e} / \sigma_{e}^{2}=\sigma_{e_{b}}^{2} / \sigma_{e}^{2}$ is the proportion of environmental variance due to among-patches differences, and $1-h^{2}=$ $\sigma_{e}^{2} / \sigma_{z}^{2}$ is the proportion of phenotypic variance in recognition traits that is due to environment.

The expectation of $\eta$ among individuals stemming from different patches equals 0 (because they share neither genes nor environment) and lies between 0 and 1 among patch mates who share some of their genes and some of their environment. If individuals could evaluate an infinite number of recognition traits, their estimate of similarity $(\eta)$ would exactly match $\bar{\eta}$ so that immigrants would be recognized unambiguously from residents. However, only a limited number of traits can be evaluated, which creates a sampling variance around $\bar{\eta}$ and makes recognition less straightforward. The sampling variance of the coefficient of regression $\eta$ of $y$ on $z$ is given by

$$
\sigma_{\eta}^{2}=\frac{1}{n}\left(\frac{\sigma_{y}^{2}}{\sigma_{z}^{2}}-\rho^{2}\right)
$$

where $n$ is the number of traits sampled and $\rho$ is the correlation coefficient between $y$ and $z$ (Lynch and Walsh 1998, their eq. [A1.20a]). Because we standardized traits so that $\sigma_{z}^{2}=\sigma_{y}^{2}=1($ and $\rho=\bar{\eta})$, this sampling variance reduces to

$$
\sigma_{\eta}^{2}=\frac{1-\bar{\eta}^{2}}{n}
$$

Figure 1 illustrates the normal distribution of perceived similarities with both immigrants $(u)$ and residents $(g)$ for different numbers of traits sampled and similarity values. As can be seen, the two distributions may overlap (some immigrants appearing more similar to the focal individual than do some residents) to the extent that few traits are sampled (fig. $1 B$ ), and similarity among patch mates is low (fig. 1C). A threshold $t$ allows the separation of two sets of potential recipients according to their estimated similarity, altruism being conceded only if recipients are more similar than $t$ to the focal individual. This occurs with probability $G_{t}$ (integral of the curve $g$ right of $t$ ) if the potential recipient is resident and with probability $U_{t}$ (integral of $u$ right of $t$ ) if it is immigrant. This threshold is one of the evolving traits in our model.
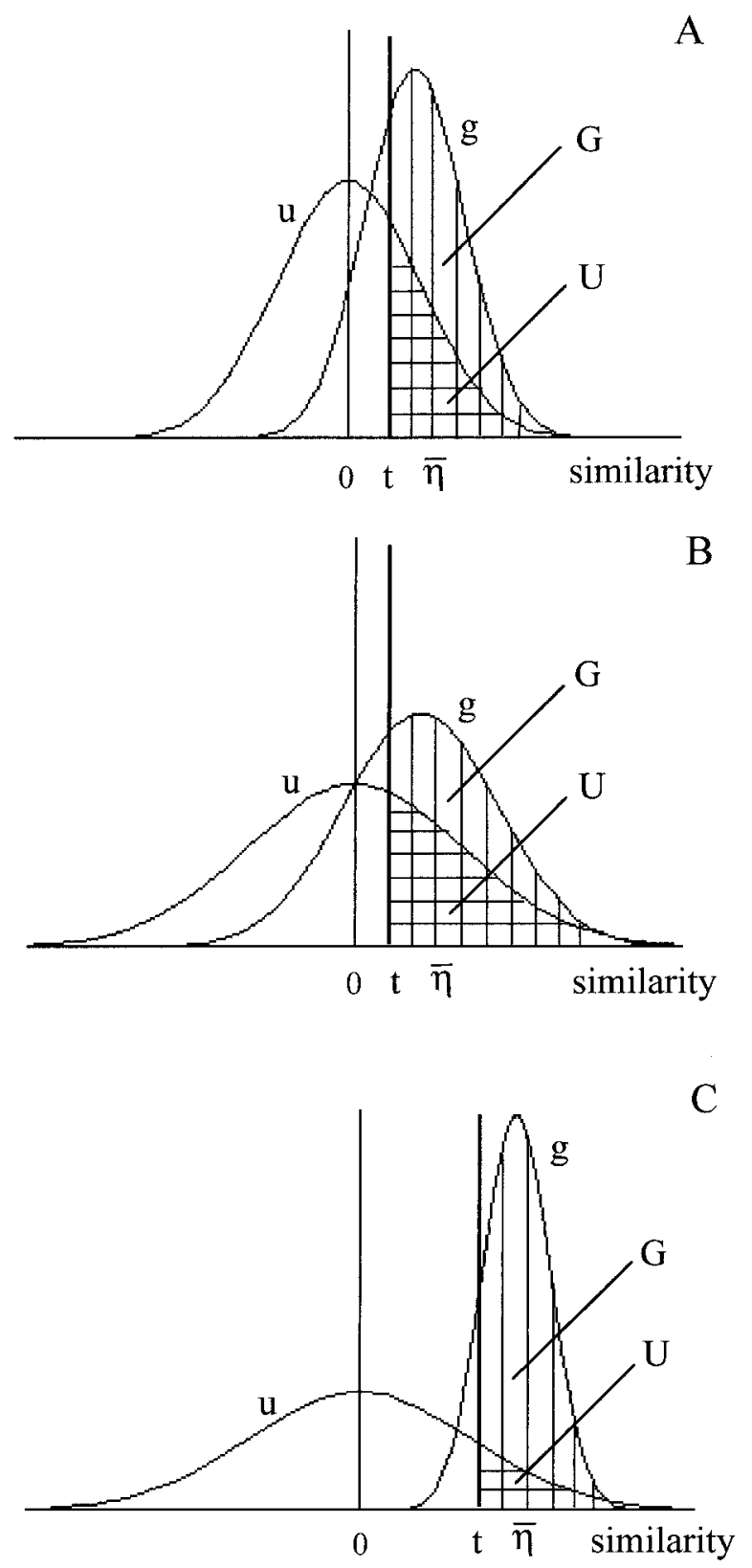

Figure 1: Distributions of similarities (as estimated by the focal individual) with immigrants ( $u$; left curve) and residents ( $g$; right curve). The former is centered on 0 and the latter on $\bar{\eta}$, the expected similarity among patch mates stemming from both common genes and common environment. Sampling recognition traits creates variance around expectations, which makes distributions overlap to some extent. A low number of recognition traits ( $n=3$ in $B$ as opposed to 10 in $A$ and $C$ ) and a low similarity among patch mates $(\bar{\eta}=0.3$ in $A$ and $B$ as opposed to 0.7 in $C$ ) increase overlap and thus lower discrimination ability. Altruism is only provided if similarity with potential recipients exceeds a threshold $t$ (bold vertical line). Thus, immigrants are accepted with probability $U$ (horizontally dashed area) and residents with probability $G$ (vertically dashed area). 


\section{Altruism}

Another trait to evolve is altruism, conceived here as in Perrin and Lehmann (2001). A level $p$ of investment is conceded, which translates into costs $C$ to the actor and benefits $B$ to the recipient, modeled in our simulations as power functions of $p$ (fig. 2). To ensure some scope for altruism, the coefficients were chosen so that marginal benefits $(b=d B / d p)$ exceed marginal costs $(c=d C / d p)$ at low investment values, while the reverse occurs at high investment values. This is to be expected whenever fitness is a saturating function of the resource traded.

\section{Direct Fitness}

Evolutionarily stable (ES) strategies for the three coevolving traits (dispersal $x$, altruism $p$, and acceptance threshold $t$ ) are obtained using the direct-fitness approach developed by Frank and Taylor (Taylor and Frank 1996; Frank 1997, 1998), writing first the fitness $w_{\bullet}$ of the focal female as a function of the traits (here, assumed to be genetically unlinked):

$$
w_{\bullet}=w\left(x_{\bullet}, x_{j}, p_{\bullet}, p_{j}, t_{\bullet}, t_{j}\right),
$$

where the traits are subscript $\bullet$ when expressed in the focal individual and subscript $j$ when expressed in females from the same patch, who share the genes of the focal female with probability $r$. This fitness function is built by noting the following:

The focal female disperses with probability $x_{\bullet}$ and survives to reach another patch with probability $s$. She then competes for $N$ breeding spots among $N m(1-x+x s)$ unrelated individuals, $m$ being the fecundity of the parental generation in an average patch. Interactions occur only with nonkin, who are mistakenly accepted with probability $U_{t} \bullet$ at cost $C_{p}$ and mistakenly accept the focal female with probability $U_{t}$, thus providing her a benefit $B_{p}$. Both costs and benefits affect her baseline fecundity $m_{0}$.

The focal female stays philopatric with probability $\left(1-x_{\bullet}\right)$, in which case she competes for $N$ breeding spots among $N\left(m_{p_{j} t_{j}}\left[1-x_{j}\right]+m x s\right)$ other females (from which $N m_{p_{j} t_{j}}\left[1-x_{j}\right]$ are also resident), $m_{p_{j} t_{j}}$ being the average fecundity of the parental generation in the focal patch (a function of their altruism and threshold).

With probability $k_{x_{j}}=\left(1-x_{j}\right) /\left(1-x_{j}+s x\right)$, this resident focal female meets a coresident and accepts her with probability $G_{t \bullet}$, thus performing altruism at cost $C_{p \bullet}$. Conversely, a resident met by the focal female will accept her with probability $G_{t}$, thereby providing her an amount of help $B_{p_{i}}$.

With probability $\tilde{k}_{x_{j}} \equiv 1-k_{x_{j}}$, the focal female interacts with an immigrant and accepts her (mistakenly) with

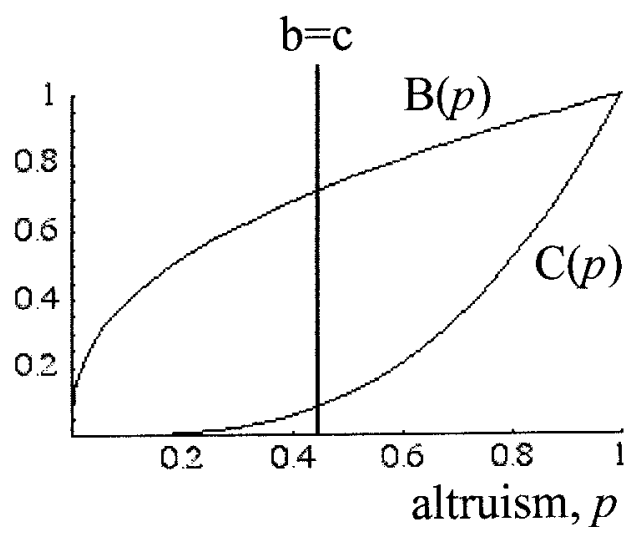

Figure 2: Benefits $(B)$ and costs $(C)$ of altruism as functions of investment $p$, here modeled as power functions $B=p^{0.4}$ and $C=p^{3}$. Values of exponents were chosen so that marginal benefits (slope of $B$ ) exceed marginal costs (slope of $C$ ) at low investment values, while the reverse occurs at high investment values. The vertical line signals the investment value for which the marginal cost to the actor equalizes the marginal benefit to the recipient $(b=c)$.

probability $U_{t \bullet}$ (which costs her $C_{p \bullet}$ ). Conversely, the immigrant makes the same error with probability $U_{t}$, thus providing her $B_{p}$.

Collecting all the above terms establishes the direct fitness function of the focal female:

$$
w_{\bullet}=\frac{\left(1-x_{\bullet}\right) m_{r}}{m_{p_{j} t_{j}}\left(1-x_{j}\right)+m x s}+\frac{x_{\bullet} s m_{i \bullet}}{m(1-x+x s)},
$$

where $\quad m_{r \bullet}=m_{0}+k_{x_{j}}\left(G_{t_{j}} B_{p_{j}}-G_{t \bullet} C_{p \bullet}\right)+\tilde{k}_{x_{j}}\left(U_{t} B_{p}-U_{t \bullet} C_{p \bullet}\right) \quad$ is her fecundity if she reproduces as a resident, and $m_{i \bullet}=$ $m_{0}+U_{t} B_{p}-U_{t} \bullet C_{p} \bullet$ is her fecundity if she reproduces as an immigrant. The corresponding values for average females are $m_{r}=m_{0}+\left(k_{x} G_{t}+\tilde{k}_{x} U_{t}\right)\left(B_{p}-C_{p}\right)$ and $m_{i}=m_{0}+$ $U_{t}\left(B_{p}-C_{p}\right)$, respectively. The productivity of the parental generation in an average patch is $m=k_{x} m_{r}+\tilde{k}_{x} m_{i}=$ $m_{0}+\left(k_{x}^{2} G_{t}+\left[1-k_{x}^{2}\right] U_{t}\right)\left(B_{p}-C_{p}\right)$, while that in the focal patch is given by $m_{p_{j} t_{j}}=m_{0}+\left(k_{x}^{2} G_{t_{j}}+\left[1-k_{x}^{2}\right] U_{t_{j}}\right)\left(B_{p_{j}}-\right.$ $C_{p_{j}}$ ). As can be seen, patch productivity increases with social interactions. Part of these benefits of altruism are exported since dispersal occurs before competition. A purely local population regulation would have prevented the emergence of altruism.

\section{Analyses}

\section{ES Altruism}

Holding the other traits $x$ and $t$ constant, the fitness function $w_{\bullet}\left(p_{\bullet}, p_{j}\right)$ is obtained from equation (4) as 


$$
\begin{gathered}
w_{p \bullet}=(1-x) \frac{m_{0}+k G\left(B_{p_{j}}-C_{p \bullet}\right)+\tilde{k} U\left(B_{p}-C_{p \bullet}\right)}{m_{p_{j}} \tilde{x}+m x s} \\
+x s \frac{m_{0}+U\left(B_{p}-C_{p \bullet}\right)}{m(\tilde{x}+x s)} .
\end{gathered}
$$

Taking the partial derivative of (5a) with respect to $p_{\bullet}$ and $p_{j}$ leads to the direct and indirect selective pressures, respectively (Frank 1998). Because these pressures are only derived in order to obtain the ESS, we only provide them at $p_{\bullet}=p_{j}=p$, which considerably simplifies notations and is always true at equilibrium. The direct selective pressure thus becomes

$$
\frac{\partial w_{p_{\bullet}}}{\partial p_{\bullet}}=\frac{-c\left[k^{2} G+\left(1-k^{2}\right) U\right]}{m},
$$

while the indirect selective pressure is

$$
\frac{\partial w_{p \bullet}}{\partial p_{j}}=\frac{k^{2}\left\{G b-\mu_{r}\left[k^{2} G+\left(1-k^{2}\right) U\right](b-c)\right\}}{m},
$$

where $c=d C / d p$ and $b=d B / d p$ designate the marginal costs and benefits of altruism (slope of the curves in fig. 2 ), and $\mu_{r}=m_{r} / m$ is the fecundity of a resident female relative to that of an average female breeding on the patch. The total selective pressure on the gene for altruism is

$$
\begin{gathered}
\frac{d w_{p \bullet}}{d p_{\bullet}}= \\
\frac{-c\left[k^{2} G+\left(1-k^{2}\right) U\right]+r\left\{k^{2} G b-k^{2} \mu_{r}(b-c)\left[k^{2} G+\left(1-k^{2}\right) U\right]\right\}}{m},
\end{gathered}
$$

where $r$ is the relatedness of the focal female to her patch mates, obtained as noted above as the regression of the breeding value of patch mates on that of the focal individual (Frank 1998). Setting this selective pressure to 0 yields the ESS level of altruism

$$
r b \gamma=c+r k^{2}(b-c) \mu_{r}
$$

where $b$ and $c$ are functions of altruism $p$ and

$$
\gamma=\frac{k^{2} G}{k^{2} G+\left(1-k^{2}\right) U}
$$

is the proportion of altruistic interactions that are not mistaken. Equation (5e) receives the intuitive interpretation that the marginal indirect benefits gained from altru- ism (left-hand side) must match the direct marginal costs (c) plus those induced by the additional kin competition stemming from altruism. It reduces to the equation (4d) of Perrin and Lehmann (2001) when perfect discrimination ( $G=1$ and $U=0$ so that $\gamma=1)$ allows altruistic acts to be always directed toward coresidents and to Perrin and Lehmann's equation (A1d) in absence of discrimination ( $G=U$ so that $\gamma=k^{2}$ and $\mu_{r}=1$ ), implying zero altruism. It is also worth noting that the selective pressure on altruism vanishes (i.e., altruism becomes a neutral character) as philopatry reaches unity. Equation (5e) is always satisfied when $k=r=1$, whatever the value of $p$.

\section{ES Dispersal}

Holding other traits ( $p$ and $t$ ) constant allows the fitness function $w_{\bullet}\left(x_{\bullet}, x_{j}\right)$ from equation (4) as

$$
\begin{aligned}
w_{x \bullet}= & \left(1-x_{\bullet}\right) \frac{m_{0}+\left(k_{x_{j}} G+\tilde{k}_{x_{j}} U\right)(B-C)}{m\left(1-x_{j}+x s\right)} \\
& +x_{\bullet} s \frac{m_{0}+U(B-C)}{m(1-x+x s)} .
\end{aligned}
$$

The direct selective pressure is obtained by the partial derivative of (6a) with respect to $x_{\bullet}$ (again calculated at $\left.x_{\bullet}=x_{j}=x\right)$ :

$$
\frac{\partial w_{x \bullet}}{\partial x_{\bullet}}=\frac{\left[m_{0}+U(B-C)\right](s-1)-k(G-U)(B-C)}{m(1-x+x s)} .
$$

The indirect selective pressure is similarly obtained by the partial derivative of (6a) with respect to $x_{j}$ :

$$
\frac{\partial w_{x} \bullet}{\partial x_{j}}=\frac{k(k-1)(G-U)(B-C)+k m_{r}}{m(1-x+x s)} .
$$

The total selective pressure on dispersal thus becomes

$$
\begin{gathered}
\frac{d w_{\bullet \bullet}}{d x_{\bullet}}= \\
\frac{(s-1)\left[m_{0}+U(B-C)\right]-k(G-U)(B-C)[1+r(1-k)]+r k m_{r} .}{m(1-x+x s)} .
\end{gathered}
$$

By setting this derivative to 0, the ES dispersal level becomes 


$$
\tilde{s}+\alpha=r[k(1+\alpha)-\tilde{k} \alpha]
$$

where $k$ is a function of dispersal $x$ and

$$
\alpha=\frac{m_{r}-m_{i}}{m_{i}}=\frac{k(G-U)(B-C)}{m_{0}+U(B-C)}
$$

is the relative gain in fecundity of residents over immigrants stemming from social interactions. Equation (6e) gets the intuitive interpretation that, at equilibrium, the inclusive costs of dispersal must match those resulting from local resource competition. Inclusive dispersal costs (left-hand side) consist of both mortality $(\tilde{s})$ plus the direct fecundity loss of not benefiting from the altruism of coresidents. Kin competition (right-hand side) also includes two terms: the breeding spot left behind by the dispersing female will be seized with probability $k$ by a related female (conducing to a relative gain in fecundity of $1+\alpha$ for this female) and with probability $1-k$ by an immigrant (so that related females will lose one opportunity of interaction and thereby lose $\alpha$ offspring).

Note that equation (6e) reduces to equation (4h) in Perrin and Lehmann (2001) when discrimination becomes perfect $(G=1$ and $U=0)$ and reduces to the classical result $(\tilde{s}=r k)$ of Frank (1986) and Taylor (1988) when discrimination is absent ( $G=U$ so that $\alpha=0$ ). Note also that the net benefits of altruism always select for philopatry. Increasing $\alpha$ weights the left-hand side of (6e) (inclusive costs of dispersal) more than the right-hand side (benefits of dispersal) unless philopatry is complete ( $r=k=1$, which cannot happen unless dispersal incurs complete mortality, $\tilde{s}=1$ ).

\section{ES Threshold}

The relevant fitness function, $w_{t}\left(t_{\bullet}, t_{j}\right)$, is obtained from equation (4) while holding all other traits $(x, p)$ constant:

$$
\begin{aligned}
w_{t \bullet}= & (1-x) \frac{m_{0}+k\left(G_{t j} B-G_{t \bullet} C\right)+\tilde{k}\left(U_{t} B-U_{t} C\right)}{m_{t_{j}} \tilde{x}+m x s} \\
& +x s \frac{m_{0}+U_{t} B-U_{t \bullet} C}{m(\tilde{x}+x s)} .
\end{aligned}
$$

The direct selective pressure on the threshold value $t$ (evaluated at $\left.t_{\bullet}=t_{j}=t\right)$ is

$$
\frac{\partial w_{t \bullet}}{\partial t_{\bullet}}=\frac{k^{2} g C+\left(1-k^{2}\right) u C}{m},
$$

while the indirect selective pressure is

$$
\frac{\partial w_{t \bullet}}{\partial t_{j}}=\frac{-k^{2} g B+k^{2} \mu_{r}\left[k^{2} g+\left(1-k^{2}\right) u\right](B-C)}{m},
$$

where $g$ and $u$ are the densities of desirable and undesirable individuals at threshold similarity $t$. The total selective pressure on the threshold value is thus

$$
\frac{d w_{t \bullet}}{d t_{\bullet}}=\frac{-r k^{2} g B+\left[C+r k^{2}(B-C) \mu_{r}\right]\left[k^{2} g+\left(1-k^{2}\right) u\right]}{m} .
$$

Setting this pressure to 0 leads to the ES threshold

$$
k^{2} g \beta=\left(1-k^{2}\right) u
$$

where

$$
\beta=\frac{r B-\left[C+r k^{2}(B-C) \mu_{r}\right]}{C+r k^{2}(B-C) \mu_{r}}
$$

is the relative net benefit of social interactions. Substituting

$$
g=\frac{1}{\sigma_{\eta} \sqrt{2 \pi}} \exp -\frac{(t-\eta)^{2}}{2 \sigma_{\eta}^{2}}
$$

(from the normal distribution) in (7e) (as well as the corresponding expression for $u$ ) provides, after some rearrangements,

$$
t=\frac{1}{\eta}-\frac{1}{n \eta} \sqrt{\left(1-\eta^{2}\right) n\left\{n-2 \ln \left[\frac{\sqrt{1-\eta^{2}}\left(1-k^{2}\right)}{k^{2} \beta_{t}}\right]\right\}} .
$$

Equation (7e) receives the intuitive interpretation that the marginal benefit of accepting a desirable recipient (lefthand side) must match the marginal cost of accepting an undesirable recipient. Or, in other words, at $t$, the ratio of mistaken $\left(\left[1-k^{2}\right] u\right)$ to correct $\left(k^{2} g\right)$ interactions must equal the relative net benefit of a social interaction. If dispersal is complete $(k=0)$ or altruism brings no benefits $(\beta=0)$, then the optimal ratio $u / g$ vanishes so that the optimal threshold tends to infinity (accept nobody). This is also directly clear from equation $(7 \mathrm{~g})$. If, by contrast, philopatry becomes complete (and provided benefits are non-null), then the threshold tends to minus infinity (accept everyone). Remember, however, that altruism becomes neutral at the limit $k=1$. 

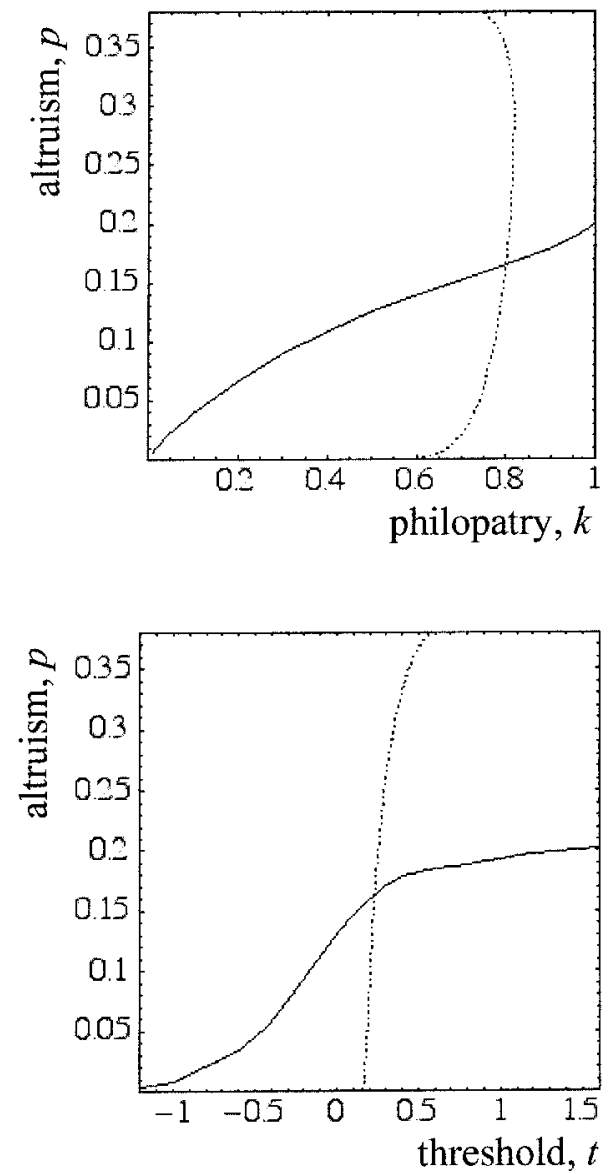

The joint optimum for the three traits must be found by simultaneous solution of the three separate ES equations. These were evaluated numerically (using Mathematica; Wolfram 1991) for a range of parameter values. Equilibrium relatedness was obtained from recurrence equations that account for the effect of patch size, dispersal rate, and differential fecundity of residents (app. B). The parameters defining the costs and benefits of altruism had fixed values in all simulations shown here (namely, $B=p^{0.4}$ and $C=p^{3}$; fig. 2). Figure 3 displays the ESSs for each of the three evolving traits (altruism $p$, philopatry $k$, and acceptance threshold $t$ ) as functions of the two others. Each two-dimensional curve represents the best response (i.e., ESS) of the variable plotted on one axis to changes imposed to the variable on the other axis, while the third variable is allowed to adapt. In the examples shown, heritability and between-group environmental variance were set to intermediate values $\left(h^{2}=e=0.5\right)$, while other parameters were fixed to $n=10, N=4, m_{0}=1$, and $s=$ 0.9 .

Figure $3 A$ shows that philopatry (dashed line) first increases, then decreases with investments in altruism. This stems from the dependence of $\alpha$ (eq. [6f] ) on the net benefit of altruism $(B-C)$ that first increases then decreases with $p$ (fig. 2). Philopatry is selected as long as altruism brings more benefits than costs, excessive investments selecting for "selfish" dispersal. In turn, altruism (solid line) responds drastically to changes in philopatry, disappearing when dispersal is complete $(k=0)$ and accruing progressively to its maximal value as philopatry tends to unity (though becoming a neutral character at the limit).

Figure $3 B$ shows strong interactions between altruism (solid line) and acceptance threshold (dashed line). A low threshold (tolerant actors) prevents altruism because many nonrelatives then become recipients, while a high threshold (intolerant actors) drives it to its maximal value because recipients have a much higher probability to be relatives. Reciprocally, the optimal threshold increases

Figure 3: Plane projections of the evolutionarily stable strategies (ESSs) for all evolving variables (altruism $p$, philopatry $k$, and acceptance threshold $t$ ). Each of the ESSs equations (5e), (6e), and (7e) define a surface in the three-dimensional $(p-k-t)$ space. Two-by-two intersections of these surfaces define curves in this three-dimensional space, the projections of which are plotted here. For example, the solid curve in $A$ is the joint

solution to (5e) and (7e) projected on the $p-k$ plane, while the dashed curve is the solution to (6e) and (7e) projected on the same plane. Parameter values were set to $N=4, n=10, m_{0}=1, h^{2}=0.5$, and $e=0.5, B=p^{0.4}$, and $C=p^{3}$. A, Evolutionarily stable (ES) altruism $p$ (solid line) increases monotonically with philopatry $k$, while ES philopatry $k$ (dashed line) first increases, then decreases with altruism $p$. B, Altruism (solid line) increases drastically with the threshold $t$, while the latter shows only a light increase with altruism. $C$, Philopatry (dashed line) is highly sensitive to changes in the threshold value, while the latter increases only slightly with the former. 
significantly as altruism is increased, which prevents high investments from being wasted on nonrelatives.

Figure $3 C$ shows the interactions between acceptance threshold and philopatry. The latter (dashed line) displays a nonmonotonic relation with $t$, stemming from its dependence on $\alpha$ (eqq. [6e]-[6f]). The function $(G-U)$ declines rapidly on each side of a maximum given by the crossing of the two bell curves (fig. 1C). By contrast, the threshold (solid line) is only slightly affected by philopatry and actually increased. This is a nontrivial result. Equation (7e) implies that the ratio $u / g$ should increase as $k$ increases so that $t$ should decrease (fig. 1). It turns out, however, that increasing $k$ also increases $r$ and $p$ (see fig. 3A), which both affect $\beta$ (eq. [7f]). This indirect effect cancels out the direct effect of $k$ over much of the range and overweigh it at large $k$ values.

For the set of parameters chosen, these best-response curves intersect at about $t=0.22, k=0.8$, and $p=$ 0.165 , which are the three-traits ESS searched (all inner equilibria discussed here were found numerically to be continuously stable).

Figure 4 shows the effect of varying dispersal cost on the evolutionarily stable philopatry $(k)$ and altruism $(p)$, for different values of heritability $\left(h^{2}\right)$ and common environment $(e)$. The main effect of dispersal costs is to favor philopatry, which, in turn, enhances altruism. However, the strength of this effect varies drastically with $h^{2}$ and $e$.

Let us first consider a situation where groups have no common environmental variance ( $e=0$; fig. $4 A)$. Low dispersal costs $(s=0.99)$ foster very high dispersal so that altruism remains extremely limited. A slight increase in costs $(s=0.95)$ boosts philopatry more than altruism. Gene flow is still too high, not only to build up significant relatedness but, more importantly, to allow good discrimination. As costs further increase, philopatry reaches values that allow more altruism to evolve but only insofar as a high heritability in recognition traits $\left(h^{2}=1\right)$ permits good discrimination. Otherwise, the large overlap in recognition traits (due to the individual component of environmental variance) prevents efficient recognition. The maximal altruism is reached as philopatry tends to unity and for traits with perfect heritability. This point corroborates Lacy and Sherman's (1983) result, which showed under similar assumptions (continuous traits with no common-environment component) that high heritability improves recognition (their fig. 8).

The introduction of a common-environment term into recognition traits $(e=0.25$; fig. $4 B$ ) changes nothing if traits have perfect heritability (because similarity in this case does not depend on $e$; eq. [2c]) but improves discrimination (thus enhancing altruism and philopatry) if traits have lower heritability. Discrimination exceeds that for high-heritability traits at high dispersal (because re-

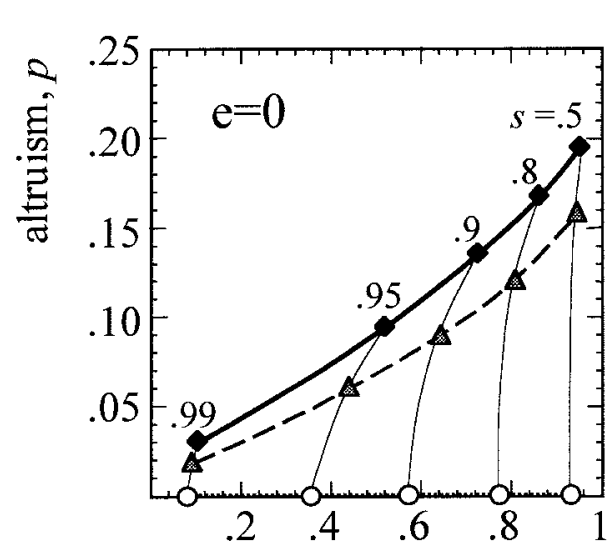

A
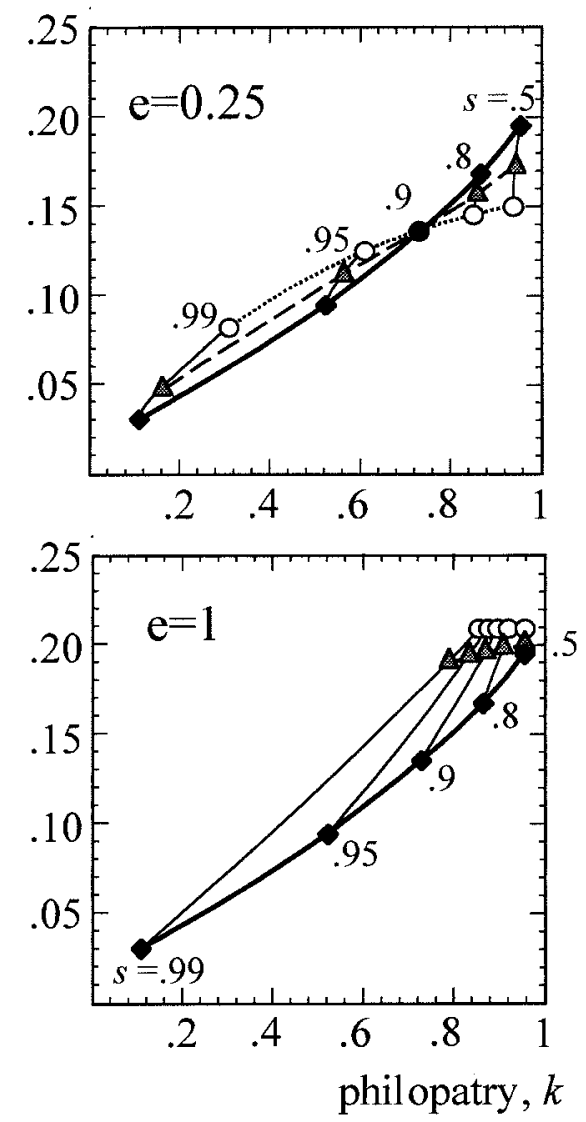

$\mathrm{C}$

Figure 4: Effects of dispersal costs on evolutionarily stable philopatry and altruism depend on the structure of recognition traits. Open circles and dotted lines, $h^{2}=0$; shaded triangles and dashed lines, $h^{2}=0.5$; filled diamonds and solid lines, $h^{2}=1$. If the common-environment component $(e)$ is low $(A)$, then the emergence of sociality requires dispersal costs and can only rely on traits with high heritability. As $e$ increases ( $B$ and $C$ ), dispersal costs lose their importance, provided cues are nonheritable. At large $e$ values $(C)$, nonheritable cues always do better, and sociality emerges independently of dispersal costs. Other parameter values: $N=4, n=10, m_{0}=1$. 
semblance among patch mates is mediated by their common environment and, thereby, is not affected by gene flow) but not at high philopatry (because similarity then stems more from common ancestry). The several curves generated by different heritability values cross at the point where discrimination is unaffected by heritability, localized at the dispersal-rate value that equalizes $r$ and $e$ (eq. [2c]).

As $e$ becomes complete $(e=1$; fig. $4 C$ ), discrimination improves drastically for low-heritability traits. The crossing of the curves now shifts totally on the right border because complete philopatry is required to induce a relatedness of 1. The best discrimination (and thus highest altruism and philopatry) are always achieved when recognition traits show no heritability. Altruism is then completely independent of dispersal costs, and philopatry shows only slight dependence because it is driven by the benefits of kin interaction much more than by the costs of dispersal.

That $e$ only matters when $h^{2}$ is low comes from obvious reasons. As environmental variation vanishes, it becomes irrelevant whether $e$ occurs within or among patches (eq. [2c]).

Figure 5 shows the effect of variations in group size. As $N$ decreases from 10 to 2, relatedness increases (eq. [B8]), which enhances both dispersal (because kin competition becomes very significant) and altruism (because recipients are more related), especially at high dispersal costs and philopatry values. The crossing of the curves shifts on the left because at small group size a lower philopatry is required for relatedness to match a given $e$ value. Thus, genetic cues should be preferred at small effective-group size and environmental cues should be preferred at large group size.

Figure 6 finally shows altruism and philopatry to depend on the number of traits examined, increasing rapidly with $n$ at low $n$ values, then leveling off. The evaluation of more traits allows the sampling variance in similarity to decrease (cf. fig. $1 A$ and $1 B$ ), which reduces strongly the overlap of distributions when variance is large but much less so when it is already low. Thus, the benefits of sampling more traits show diminishing returns. If there were any costs to the sampling process (not implemented here), then the optimal number of traits to be sampled could be inferred (and would presumably depend on both $h^{2}$ and $e$ ).

\section{Discussion}

Discrimination ability plays a key role in the evolution of sociality by promoting altruism through the decoupling of social and economic neighborhoods. Errors in kin recognition, therefore, lower the optimal investments in altruism and thereby lower the benefits expected from philopatry and kin interactions. This has the potential to impede the evolution of social structures. The conse-
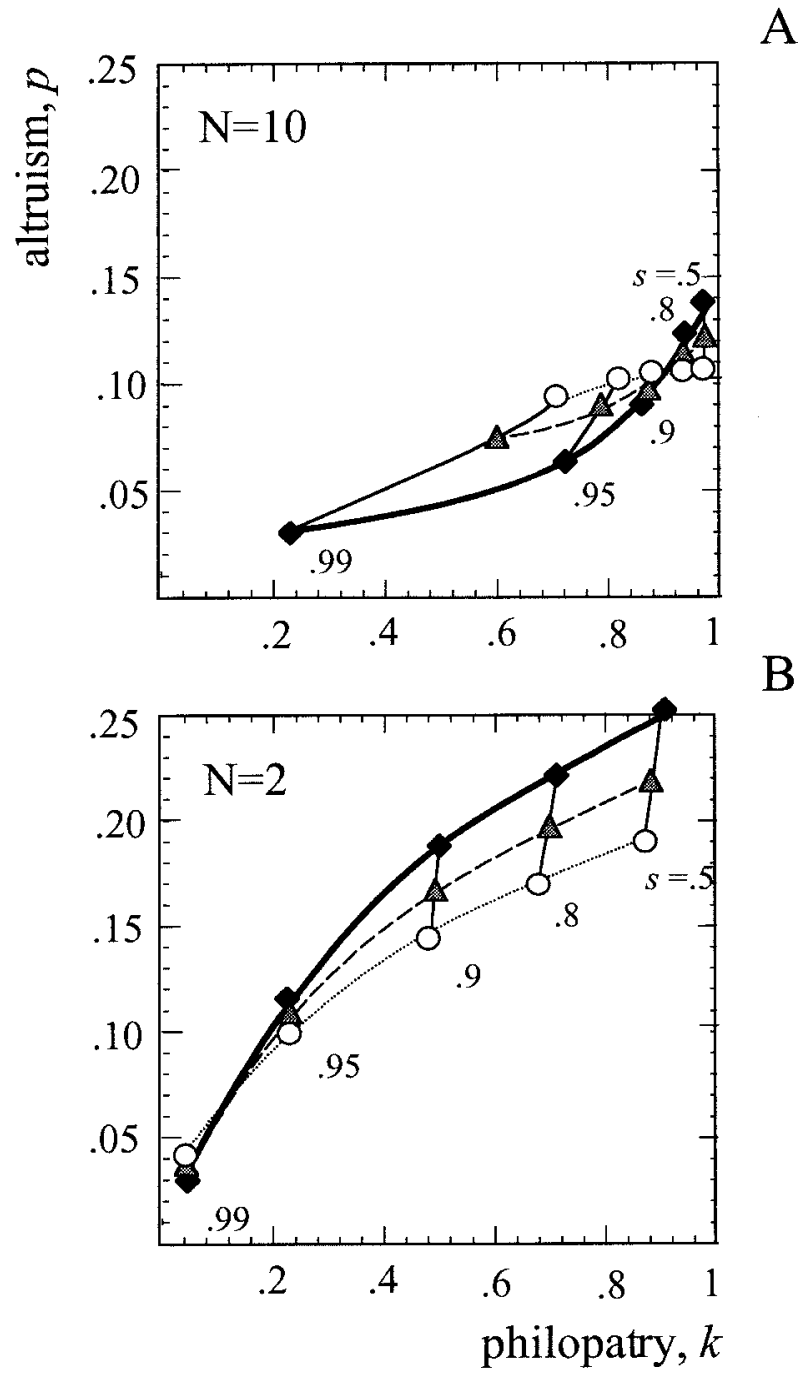

Figure 5: Effective population size $(N)$ affects the consequences of dispersal costs on evolutionarily stable philopatry and altruism. Symbols as in figure 4. At small $N$ values $(N=2$ in $B$ vs. 10 in $A$ ), high relatedness boosts altruism, and also dispersal (because of kin competition), so that varying group size produces a negative correlation between altruism and philopatry. High relatedness (small $N$ ) furthermore provides advantages to genetic cues. Other parameter values: $e=0.25, n=10, m_{0}=1$.

quences, developed below, are first that the costs of dispersal (as opposed to the benefits of philopatry) play a major role in promoting sociality when discrimination is low but much less so when discrimination is high. Second, not only should natural selection favor an optimal acceptance threshold but also the use of cues that maximize discrimination.

\section{Dispersal Costs and Sociality}

Perrin and Lehmann (2001) found that ecological constraints are required for the emergence of altruism if kin 

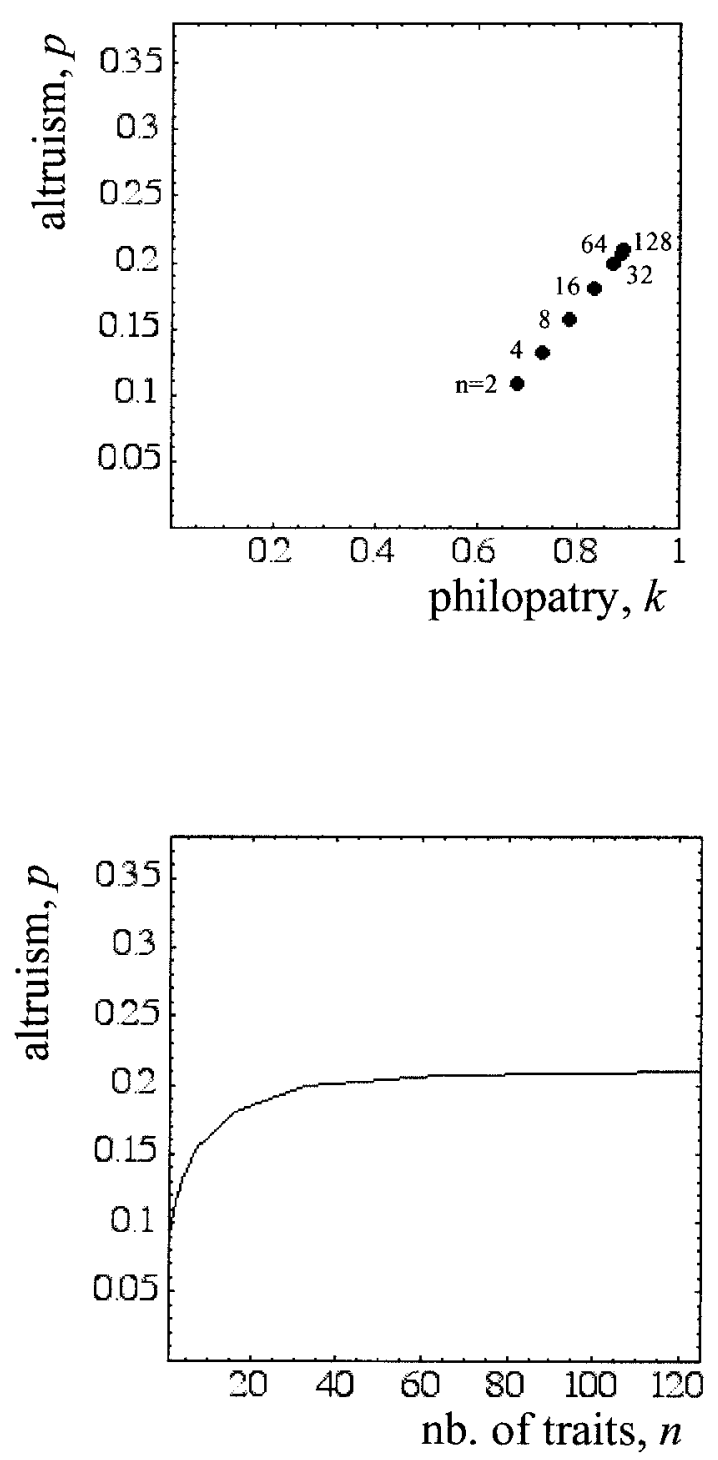

Figure 6: $A$, A large number of recognition traits $(n)$ increase philopatry, so that $n$ induces a positive correlation between altruism and philopatry. $B$, For a given expected similarity $\bar{\eta}$, the amount of altruism conceded $(p)$ increases with the number of recognition traits $(n)$ because of the concomitant reduction in sampling variance (see fig. 1) but at a decelerating pace (negative second derivative).

discrimination is spatially based. In contrast, associative learning may favor altruism even in complete absence of dispersal costs. The situation investigated here shows a continuum between these two outcomes. In the case of perfect discrimination $\left(h^{2}=0, e=1, n\right.$ is large), dispersal costs have no effect whatsoever on the equilibrium altruism value and only a slight effect on philopatry because the benefits of altruism suffice to counteract the costs of kin competition (fig. 4C). As discrimination worsens (fig. $4 A$ ), costs become more and more important in preventing kin competition to drive complete dispersal. This result confirms that kin-discrimination ability has a significant role to play in the debate as to whether sociality is driven by the costs of dispersal or the benefits of philopatry (Stacey and Ligon 1991). An empirically relevant corollary is the negative correlation expected between the cognitive capacities of organisms and the importance of ecological constraints in molding social structures.

Another potentially interesting empirical prediction stemming from the patterns documented here is that sociality is likely to be maintained by high dispersal costs in those species that use genetic cues for kin discrimination. Indeed, our analysis shows (fig. 4) that high dispersal costs are always required for sociality to emerge if recognition traits have a high heritability.

\section{Optimal Acceptance Threshold}

B The situation investigated here most closely matches Reeve's (1989, p. 417) "frequency-dependent context with pair-wise kin interactions." Introducing his first-order condition (12) into his fitness equation (11) provides the equilibrium condition

$$
\frac{u}{g}=\frac{k}{1-k} \frac{r B-C}{C}
$$

(using our symbols and our assumption that relatedness with undesirable recipients is 0 ). Although the global interpretation is similar (at equilibrium, the marginal benefits of accepting a desirable recipient match the marginal costs of accepting an undesirable one), this equation differs from ours (7e) on two points. First, we obtain $k^{2}$ and $1-k^{2}$ instead of $k$ and $1-k$ because we also include the possibility that the focal individual may end up as an immigrant. Second, we integrate kin competition, which adds further costs to altruism. But more importantly, the accounting of dynamic interactions introduces important indirect effects that drastically affect the expected outcome.

First, as already noted, the frequency of interactions with desirable recipients $(k)$ increases $t$ (fig. $3 C$ ) rather than decreasing it because of indirect effects on the relative net benefit of social interactions $(\beta)$. Similarly, though the optimal threshold is indeed expected to decrease as the benefits of altruism increase (Reeve 1989), it happens to increase with altruistic investments (fig. $3 B$ ) because local competition renders misguided investments very costly. Increasing the threshold prevents investments from being wasted on unrelated recipients.

Relatedness with desirable recipients also shows complex interactions. On the one hand, it increases the benefits 
of altruism (thereby selecting for a lower threshold), but on the other hand, it increases similarity with desirable recipients (eq. [2c]), thereby selecting for a higher threshold (eq. $[7 \mathrm{~g}]$ ). The resulting balance is bound to depend on the heritability of recognition traits since a low heritability would cancel the effect of relatedness on similarity. Finally, the number of traits investigated $(n)$ is shown to affect the threshold (eq. $[7 \mathrm{~g}]$ ) by reducing the sampling variance of similarities.

\section{Genetic versus Environmental Cues}

Lacy and Sherman (1983) showed that when similarity between kin is solely the result of additive genetic variation then increasing cue heritability improves recognition. They did not investigate environmental cues but suggested these might reduce kin recognition errors if environment affects the phenotypes of kin classes differentially. According to our results, the best kin discrimination (and thereby the highest altruism and philopatry) is indeed achieved by using recognition traits with low heritability and a high between-group component of environmental variance (fig. 4C). This combination boosts similarity among patch mates (eq. [2c]) at gene-flow values that would otherwise induce much overlap in heritable traits. High $\eta$ values not only drive apart the expectations of similarity distributions for residents and immigrants but also reduce their sampling variance (eq. [3]), which contributes to improve discrimination.

Though the cuticular hydrocarbon profiles of honeybees possess enough discriminating power to allow for genetically based kin recognition (Arnold et al. 1996), guards use exclusively environmental cues acquired in the adult stage when encountering entering bees (Downs and Ratnieks 1999). (Whether genetic cues are used at all for patrilineal recognition within hives remains a disputed issue; Breed et al. 1994). Environmental similarity in odors among nest mates in social insects has been shown to stem either from the use of common sources of food or from contact with the common-nest material (Crosland 1989a). Aggressiveness among colonies of the leaf-cutting ant $A c$ romyrmex octospinosus drops when they are fed the same food (Jutsum et al. 1979). Honeybee colonies or sister groups fed the same flavored syrup accept transferred workers but reject workers fed syrup of a different flavor (references in Downs and Ratnieks 1999). The peculiar blend of plants (dictated by colony environment) used by colonies of Polistes fuscatus wasps to build their nest durably impregnate bee nymphs with a colony-specific odor (Gamboa et al. 1996). Waxcomb plays a similar role in honeybees (Breed at al. 1988, 1995), thus allowing nestmate recognition to develop within a few days of colony swarming (Breed et al. 1998).
However, traits with high heritability become preferable as soon as relatedness exceeds the between-group component of environmental variance. This may arise when severe dispersal costs induce a strong philopatry (fig. 4B) or when small group size boosts relatedness (fig. 5). By group size, we refer here to the effective number of breeders, not to the actual number of individuals. Ant colonies with one singly mated queen obviously belong in this category, whatever the number of workers. Relatedness among sisters in this case theoretically reaches values as high as 0.75 (due to the haplodiploid sex-determining system of hymenopterans), which presumably exceeds the between-group environmental component of most phenotypic traits. Highly heritable recognition traits are likely to be chosen in this case.

Rare alleles allow better discrimination. Frequencydependent selection acting in large populations should thus maintain significant heritabilities in recognition traits over evolutionary times (Ratnieks 1991). However, the drop in genetic variance following population bottlenecks may generate unexpected side effects. Unicoloniality in the invading Argentine ant (Linepithema humile) apparently results from the loss of genetic diversity in recognition traits due to founder effects (Tsutsui et al. 2000). Natural populations in native habitats show a high genetic diversity, high discrimination ability, and high relatedness among nest mates, contrasting drastically with unicolonial invading populations. According to our results, however, the drop in relatedness among nest mates may confer advantages to environmental cues (fig. $4 B$ ). An evolutionary shift to such cues would, in turn, restore multicoloniality. Whether unicoloniality in invading ants is indeed a transient state due to the breakdown of recognition systems is a fascinating hypothesis that deserves continued empirical investigation.

Empirical evidence suggests that recognition cues usually lay in a continuum between purely genetic and primarily environmental (Ratnieks 1991), which presumably arises from the balance between several interacting factors. In addition to small effective size and high dispersal costs (both of which favor genetic cues), cognitive ability should matter. The evolved brains of higher vertebrates should facilitate the transmission and interpretation of nongenetic information. The structure of environmental variance is obviously also of prime importance. Environmental cues should be favored in coarse-grained habitats showing most heterogeneity at a large spatial scale (among patches), while genetic cues might be preferred in homogeneous or fine-grained habitats, in which important small-scale heterogeneity may amplify individual differences (withinpatch variance) for environmentally determined phenotypic traits.

However, the possibility of an active manipulation of 
the variance components of recognition traits has also to be considered. Any decrease in individual (within group) environmental variance will enhance $e$ (proportion of environmental variance that is due to differences between groups), thus improving discrimination and altruism. This may be achieved by standardization of recognition traits among patch mates, either through individual adoption of a common standard or by mixing up and sharing of cues at the group level. Trophallaxis in social insects may also serve the purpose of homogenizing food-mediated environmental cues (Dahbi et al. 1999). Nest mates in several ant species have been shown to exchange and share cuticular hydrocarbons through mutual licking and passive contact (Soroker et al. 1994, 1995; Vienne et al. 1995). By reducing the interindividual component of environmental variance, these exchanges create a colony-specific odor, the so-called gestalt chemical signature (Crozier and Dix 1979; Stuart 1987, 1988; Crosland 1989b; Tsuji 1990; Dahbi and Lenoir 1998a, 1998b). Only the environmental component of recognition traits is of course affected in the process, not their genetic part (relatedness is unchanged). Albeit individually produced odors might be under complete genetic control, the odor of nest mates is definitely part of the environment. Similar mixing of individual odors to form a unique recognition cue has been found in bees (Breed and Julian 1992) and in naked mole-rats (O'riain and Jarvis 1997). Polydomous ant colonies may also exchange workers to maintain a uniform odor over multiple nests (Dahbi et al. 1997).

In social vertebrates, vocalization convergence within social units may play a similar function. Sonar signals of big brown bats (Eptesicus fuscus) contain information about family affiliation (Masters et al. 1995). Acoustic convergence within social groups and differences between groups in greater spear-nosed bats (Phyllostomus hastatus) arise through vocal learning (Boughman 1998). Flockspecific call differences observed in black-capped chickadees arise through a process of mutual imitation (Nowicki 1989). Males of the cooperatively breeding stripe-backed wren (Campylorhynchus nuchalis) learn repertoires of stereotyped calls from older male relatives. As a result, these vocalizations are normally specific to patrilineal family groups (Price 1999). A similar process may occur in humans, who show an impressive propensity to comply with the cultural peculiarities of the group they are born in, in terms of language (dialects and accents), habits, manners, and customs as well as ornaments (tribal clothes, ritual paintings or tattoos; Cordell and Schwartz 1979). Cultural adornments take an important discrimination function as soon as contacts among distinct communities become frequent (Delaporte 1990). Genetically based recognition traits would definitely not allow easy discrimination on small geographical scales. Human meticulous compliance with social standards might thus have been partly selected for recognition purpose through its efficient increase of the between-group component of environmental variance and concomitant decrease in within-group variance.

\section{Acknowledgments}

This work was supported by the grants 31-38762.93 and 31-59442.99 from the Swiss National Science Foundation. We thank F. Balloux, M. Chapuisat, E. Petit, and M. Reuter for discussions and comments on an earlier draft. We are indebted to S. Aron, J. Herbers, J. Lucas, A. Maeder, and L. Passera for sharing their knowledge of the literature on social insects, birds, and mammals. 


\section{APPENDIX A}

Table A1: Symbols used in the model

\begin{tabular}{|c|c|}
\hline Symbol & Definition \\
\hline$x$ & Dispersal probability \\
\hline$p$ & Investment in altruism \\
\hline$t$ & Similarity threshold above which potential recipients are accepted \\
\hline$s$ & Survival probability during dispersal \\
\hline$N$ & Number of breeding females per patch \\
\hline$k_{x_{j}} \equiv \tilde{x}_{j} /\left(\tilde{x}_{j}+s x\right)$ & Probability that an individual breeding in the focal patch was born locally \\
\hline$C_{p}$ & Fecundity cost of providing altruism, an accelerating function of $p$ (fig. 2) \\
\hline$B_{p}$ & Fecundity benefit of receiving altruism, a decelerating function of $p$ (fig. 2) \\
\hline$e$ & Proportion of the environmental variance in phenotypic traits due to differences among groups \\
\hline$h^{2}$ & Heritability of a trait (proportion of phenotypic variance that is additive genetic) \\
\hline $\bar{\eta}$ & Expectation of phenotypic similarity among patch mates \\
\hline$g_{\tau}$ & $\begin{array}{l}\text { Probability that a female born in the same patch as the focal one has similarity } \tau \text { to her. } \\
\text { Similarity may range minus infinity to plus infinity, though its expectation }(\bar{\eta}) \text { ranges } 0-1 \text { (fig. } 1 \text { ) }\end{array}$ \\
\hline$u_{\tau}$ & $\begin{array}{l}\text { Probability that a female stemming from a different patch than the focal one displays a } \\
\text { similarity } \tau \text { to her (fig. 1) }\end{array}$ \\
\hline$G_{t} \equiv \int_{\tau=t}^{\infty} g_{\tau} d \tau$ & Probability of accepting a desirable recipient, function of the threshold $t$ \\
\hline$U_{t} \equiv \int_{\tau=t}^{\infty} u_{\tau} d \tau$ & Probability of accepting an undesirable recipient \\
\hline$m_{o}$ & Baseline fecundity of a breeding female (fecundity in absence of social interaction) \\
\hline$m_{r}, \sigma_{r}^{2}$ & Average and variance in the progeny of a breeding resident female \\
\hline$m_{i}, \sigma_{i}^{2}$ & Average and variance in the progeny of a breeding immigrant female \\
\hline$m \equiv k m_{r}+\tilde{k} m_{i}$ & Average fecundity per female in the whole metapopulation \\
\hline$\mu_{r} \equiv m_{r} / m$ & Relative fecundity of a resident \\
\hline$\mu_{i} \equiv m_{i} / m$ & Relative fecundity of an immigrant \\
\hline$\alpha \equiv\left(m_{r}-m_{i}\right) / m_{i}$ & Relative gain in progeny of residents over immigrants \\
\hline$\theta$ & $\begin{array}{l}\text { Average coancestry among individuals born on the same patch (probability that alleles sampled } \\
\text { from two individuals are identical by descent) }\end{array}$ \\
\hline$F=k^{2} \theta \mu_{r}$ & Average inbreeding (probability that an individual bears two alleles identical by descent) \\
\hline$r=2 \theta /(1+F)$ & $\begin{array}{l}\text { Average relatedness among individuals born on the same patch, equal to the coancestry among } \\
\text { them divided by coancestry with self }\end{array}$ \\
\hline$P_{i}$ & Probability that two offspring share a same parent of sex $i$ \\
\hline$Q_{i j}$ & $\begin{array}{l}\text { Probability that the parent }(\operatorname{sex} i) \text { of a random offspring and the parent }(\operatorname{sex} j) \text { of another random } \\
\text { offspring were different individuals but both resident }\end{array}$ \\
\hline
\end{tabular}

Note: Traits have subscript dot when expressed in the focal individual. Subscript $j$ designates average value of the trait in individuals from the same patch as the focal female (and bearing with her relatedness $r$ ), and absence of subscript dot denotes the average value for the whole metapopulation. Tilde denotes the complement to unity (e.g., $\tilde{P}_{m} \equiv 1-P_{m}$ ).

\section{APPENDIX B}

\section{Dynamics of Relatedness}

\section{General Equations}

Our recurrence equation is derived along the lines proposed by Nagylaki (1995) and Wang (1997). The coancestry $\left(\theta_{t+1}\right)$ between two offspring born on the same patch is the probability that two alleles randomly sampled, one from each offspring, are identical by descent (IBD). 
First, with probability one-fourth, both alleles were inherited along the paternal lines. If the offspring share the same father, then IBD probability is $\left(1+F_{t}\right) / 2$. If fathers differ, then IBD probability equals their coancestry. Under infiniteisland assumptions, this means $\theta_{t}$ if both fathers were born locally and 0 otherwise. Thus, paternal descent ensures a coancestry of

$$
\frac{1}{4}\left(P_{\mathrm{m}} \frac{1+F_{t}}{2}+Q_{\mathrm{mm}} \theta_{t}\right)
$$

where $P_{\mathrm{m}}$ is the probability that two random offspring share the same father and $Q_{\mathrm{mm}}$ is the probability that their fathers differed, but both were resident.

Second, with probability one-fourth, both alleles were inherited from mothers. Using the same reasoning, maternal descent ensures a coancestry of

$$
\frac{1}{4}\left(P_{\mathrm{f}} \frac{1+F_{t}}{2}+Q_{\mathrm{ff}} \theta_{t}\right)
$$

where $P_{\mathrm{f}}$ is the probability that two random offspring share the same mother and $Q_{\mathrm{ff}}$ is the probability that their mothers differed, but both were resident.

Finally, with probability one-half, these alleles were inherited, one from a mother and one from a father. The IBD probability in that case equals the expected coancestry among these parents, $Q_{\mathrm{fm}} \theta_{t}$, where $Q_{\mathrm{fm}}$ measures the probability that a random male and a random female were both born locally.

Collecting all these terms provides the recurrence equation for coancestry:

$$
\theta_{t+1}=\frac{1}{4}\left[\left(P_{\mathrm{m}}+P_{\mathrm{f}}\right)\left(\frac{1+F_{t}}{2}\right)+\left(Q_{\mathrm{mm}}+Q_{\mathrm{ff}}+2 Q_{\mathrm{fm}}\right) \theta_{t}\right]
$$

Since $r=2 \theta /(1+F)$, dividing both sides by $\left(1+F_{t}\right) / 2$ provides the corresponding equation for relatedness. At equilibrium $\left(r_{t+1}=r_{t}=r\right)$, we thus obtain

$$
r=\frac{P_{\mathrm{m}}+P_{\mathrm{f}}}{4-Q_{\mathrm{mm}}-Q_{\mathrm{ff}}-2 Q_{\mathrm{fm}}}
$$

\section{Specific Relationships}

Assuming similar dispersal rates by males and females, the $\mathrm{Q}_{i j}$ probabilities are given by

$$
\begin{aligned}
Q_{\mathrm{fm}} & =\frac{m_{r}}{m} k^{2}, \\
Q_{\mathrm{mm}} & =k \frac{N k-1}{N}, \\
Q_{\mathrm{ff}} & =\frac{N k\left[m_{r}^{2}(N k-1)-\sigma_{r}^{2}\right]}{N m(N m-1)} .
\end{aligned}
$$

Under random mating, the probability that two offspring share the same father is

$$
P_{\mathrm{m}}=\frac{1}{N}
$$


For females, the relation is more complex since different categories of mothers (immigrants and residents) have different fecundities. The corresponding probability is thus a sum over several categories:

$$
P_{\mathrm{f}}=\pi_{i} N \tilde{k}+\pi_{r} N k
$$

where

$$
\pi_{i}=\frac{\sigma_{i}^{2}+m_{i}\left(m_{i}-1\right)}{N m(N m-1)}
$$

is the probability that two random offspring share one particular immigrant mother and

$$
\pi_{r}=\frac{\sigma_{r}^{2}+m_{r}\left(m_{r}-1\right)}{N m(N m-1)}
$$

is the corresponding probability for a resident mother. From the binomial distribution, the variances in the progeny of immigrant and resident mothers are $\sigma_{i}^{2}=m_{i}\left[1-\left(m_{i} / N m\right)\right]$ and $\sigma_{r}^{2}=m_{r}\left[1-\left(m_{r} / N m\right)\right]$, respectively. Substituting these values into (B7a), (B7b), and (B6) provides $\pi_{i}=\left(\mu_{i} / N\right)^{2}, \pi_{r}=\left(\mu_{r} / N\right)^{2}$, and $P_{\mathrm{f}}=(1 / N)\left(k \mu_{r}^{2}+\tilde{k} \mu_{i}^{2}\right)$. Equation (B2) thus becomes

$$
r=\frac{1+k \mu_{r}^{2}+\tilde{k} \mu_{i}^{2}}{4 N-k[(N k-1) /(N-1)]\left(2 N-1-k \mu_{r}^{2}-\tilde{k} \mu_{i}^{2}\right)-2 k^{2} N} .
$$

If resident and immigrant females have the same fecundity $\left(\mu_{r}=\mu_{i}=1\right)$, then (B8) reduces to the basic equation for randomly mating dioecious species:

$$
r=\frac{1}{2 N\left(1-k^{2}\right)+k}
$$

\section{Literature Cited}

Adams, E. S. 1991. Nest-mate recognition based on heritable odors in the termite Microcerotermes arboreus. Proceedings of the National Academy of Sciences of the USA 88:2031-2034.

Arnold, G., B. Quenet, J.-M. Cornuet, C. Masson, B. De Schepper, A. Estoup, and P. Gasqui. 1996. Kin recognition in honeybees. Nature 379:498.

Beye, M., P. Neumann, M. Chapuisat, P. Pamilo, and R. F. A. Moritz. 1998. Nestmate recognition and the genetic relatedness of nests in the ant Formica pratensis. Behavioral Ecology and Sociobiology 43:67-72.

Boughman, J. W. 1998. Vocal learning by greater spearnosed bats. Proceedings of the Royal Society of London B, Biological Sciences 265:227-233.

Breed, M. D., and G. E. Julian. 1992. Do simple rules apply in honey-bee nestmate discrimination? Nature 357: 685-686.

Breed, M. D., K. R. Williams, and J. H. Fewell. 1988. Comb wax mediates the acquisition of nest-mate recognition cues in honey bees. Proceedings of the National Academy of Sciences of the USA 85:8766-8769.
Breed, M. D., C. K. Welch, and R. Cruz. 1994. Kin discrimination within honey bee (Apis mellifera) colonies: analysis of the evidence. Behavioural Processes 33: 25-40.

Breed, M. D., M. F. Garry, A. N. Pearce, B. E. Hibbard, L. B. Bjostad, and R. E. Page. 1995. The role of wax comb in honey bee nestmate recognition. Animal Behaviour 50:489-496.

Breed, M. D., E. A. Leger, A. N. Pearce, and Y. J. Wang. 1998. Comb wax effects on the ontogeny of honey bee nestmate recognition. Animal Behaviour 55:13-20.

Cordell, J. M., and R. A. Schwartz. 1979. The fabrics of culture: the anthropology of clothing and adornments. Mouton, The Hague.

Crosland, M. W. J. 1989a. Kin recognition in the ant Rhytidoponera confusa. I. Environmental odour. Animal Behaviour 37:912-919.

- 1989 b. Kin recognition in the ant Rhytidoponera confusa. II. Gestalt odour. Animal Behaviour 37: 920-926.

Crozier, R. H. 1986. Genetic clonal recognition abilities in 
marine invertebrates must be maintained by selection for something else. Evolution 40:1100-1101.

Crozier, R. H., and M. W. Dix. 1979. Analysis of two genetic models for the innate components of colony odor in social Hymenoptera. Behavioral Ecology and Sociobiology 18:105-115.

Dahbi, A., and A. Lenoir. 1998a. Nest separation and the dynamics of the Gestalt odor in the polydomous ant Cataglyphis iberica (Hymenoptera, Formicidae). Behavioral Ecology and Sociobiology 42:349-355.

-1998b. Queen and colony odour in the multiple nest ant species, Cataglyphis iberica (Hymenoptera, Formicidae). Insectes Sociaux 45:301-313.

Dahbi, A., X. Cerda, A. Hefetz, and A. Lenoir. 1997. Social transport in the ant Cataglyphis iberica: a means to maintain a uniform colonial odour in a species with multiple nests. Physiological Entomology 22:13-19.

Dahbi, A., A. Hefetz, X. Cerda, and A. Lenoir. 1999. Trophallaxis mediates uniformity of colony odor in Cataglyphis iberica ants (Hymenoptera, Formicidae). Journal of Insect Behavior 12:559-567.

Delaporte, Y. 1990. Le vêtement dans les sociétés traditionelles. Pages 961-1031 in J. Poirier, ed. Histoire des moeurs. Gallinard, Paris.

Downs, S. G., and F. L. W. Ratnieks. 1999. Recognition of conspecifics by honeybee guards uses nonheritable cues acquired in the adult stage. Animal Behaviour 58: 643-648.

Frank, S. A. 1986. Dispersal polymorphism in subdivided populations. Journal of Theoretical Biology 122:303-309.

- 1997. Multivariate analysis of correlated selection and kin selection, with an ESS maximization method. Journal of Theoretical Biology 189:307-316.

- 1998. Foundations of social evolution. Princeton University Press, Princeton, N.J.

Gamboa, G. J., H. K. Reeve, D. Ferguson, and T. K. Wacker. 1986. Nestmate recognition in social wasps: the origin and acquisition of recognition odors. Animal Behaviour 34:685-695.

Gamboa, G. J., A. T. Grudzien, K. E. Espelie, and E. A. Bura. 1996. Kin recognition pheromones in social wasps: combining chemical and behavioural evidence. Animal Behaviour 51:625-629.

Greenberg, L. 1979. Genetic component of bee odor in kin recognition. Science (Washington, D.C.) 206:1095-1097.

Grosberg, R. K. 1988. The evolution of allorecognition specificity in clonal invertebrates. Quarterly Review of Biology 63:377-412.

Grosberg, R. K., and J. F. Quinn. 1986. The genetic control and consequences of kin recognition by the larvae of a colonial marine invertebrate. Nature 322:456-459

Hamilton, W. D. 1964. The genetical evolution of social behaviour. I, II. Journal of Theoretical Biology 7:1-16, 17-52.

Hamilton, W. D., and R. M. May. 1977. Dispersal in stable habitats. Nature 269:578-581.

Hauber, M. E., and P. W. Sherman. 2000. The armpit effect in hamster kin recognition. Trends in Ecology \& Evolution 15:349-350.

Heth, G., J. Todbrank, and R. E. Johnston. 1998. Kin recognition in golden hamsters: evidence for phenotype matching. Animal Behaviour 56:409-417.

Joseph, S. B., W. E. Snyder, and A. J. Moore. 1999. Cannibalizing Harmonia axyridis (Coleoptera: Coccinellidae) larvae use endogenous cues to avoid eating relatives. Journal of Evolutionary Biology 12:792-797.

Jutsum, A. R., T. S. Saunders, and J. M. Cherrett. 1979. Intraspecific aggression in the leaf-cutting ant Acromyrmex octospinosus. Animal Behaviour 27:839-844.

Lacy, R. C., and P. W. Sherman. 1983. Kin recognition by phenotype matching. American Naturalist 121:489-512.

Linsenmair, K. E. 1987. Kin recognition in subsocial arthropods, in particular in the desert isopod Hemilepistus reaumuri. Pages 411-436 in D. J. C. Fletcher and C. D. Michener, eds. Kin recognition in animals. Wiley, Chichester.

Lynch, M., and B. Walsh. 1998. Genetics and analysis of quantitative traits. Sinauer, Sunderland, Mass.

Manning, C. J., E. K. Wakeland, and W. K. Potts. 1992. Communal nesting patterns in mice implicate $\mathrm{MHC}$ genes in kin recognition. Nature 360:581-583.

Masters, W. M., K. A. S. Raver, and K. A. Kazial. 1995. Sonar signals of big brown bats, Eptesicus fuscus, contain information about individual identity, age and family affiliation. Animal Behaviour 50:1243-1260.

Mateo, J. M., and R. E. Johnston. 2000. Kin recognition and the "armpit effect": evidence of self-referent phenotype matching. Proceedings of the Royal Society of London B, Biological Sciences 267:695-700.

Moritz, R. B., and E. Hillesheim. 1990. Trophallaxis and genetic variance of kin recognition in honey bees, Apis mellifers L. Animal Behaviour 40:641-647.

Nagylaki, T. 1995. The inbreeding effective population number in dioecious populations. Genetics 139:473-485.

Nowicki, S. 1989. Vocal plasticity in captive black-capped chickadees: the acoustic basis and rate of call convergence. Animal Behaviour 37:64-73.

Obin, M. S. 1986. Nestmate recognition cues in laboratory and field colonies of Solenopsis invicta Buren (Hymenoptera: Formicidae): effect of environment and role of cuticular hydrocarbons. Journal of Chemical Ecology 12:1965-1975.

O'riain, M. J., and J. U. M. Jarvis. 1997. Colony member recognition and xenophobia in the naked mole rat. Animal Behaviour 53:487-498. 
Perrin, N., and L. Lehmann. 2001. Is sociality driven by the costs of dispersal or the benefits of philopatry? a role for kin-discrimination mechanisms. American Naturalist 158:471-483.

Pfennig, D. W., P. W. Sherman, and J. P. Collins. 1994. Kin recognition and cannibalism in polyphenic salamanders. Behavioral Ecology 5:225-232.

Potts, W. K., C. J. Manning, and E. K. Wakeland. 1991. Mating patterns in semi-natural populations of mice influenced by MHC genotype. Nature 352:619-621.

Price, I. J. 1999. Recognition of family-specific calls in stripe-backed wrens. Animal Behaviour 57:483-492.

Queller, D. C. 1992. Does population viscosity promote kin selection? Trends in Ecology \& Evolution 7:322-324.

. 1994. Genetic relatedness in viscous populations. Evolutionary Ecology 8:70-73.

Ratnieks, F. L. W. 1991. The evolution of genetic odorcue diversity in social Hymenoptera. American Naturalist 137:202-226.

Reeve, H. K. 1989. The evolution of conspecific acceptance thresholds. American Naturalist 133:407-435.

Soroker, V., C. Vienne, E. Nowbahari, and A. Hefetz. 1994. The postpharyngeal gland as a "gestalt" organ for nestmate recognition in the ant Cataglyphis niger. Naturwissenschaften 81:510-513.

Soroker, V., C. Vienne, and A. Hefetz. 1995. Hydrocarbon dynamics within and between nestmates in Cataglyphis niger (Hymenoptera, Formicidae). Journal of Chemical Ecology 21:365-378.

Stacey, P., and J. D. Ligon. 1991. The benefits-of-philopatry hypothesis for the evolution of cooperative breeding: variation in territory quality and group size effect. American Naturalist 137:831-846.

Stuart, R. J. 1987. Individual workers produce colonyspecific nestmate recognition cues in the ant, Leptothorax curvispinosus. Animal Behaviour 35:1062-1069.
1988. Collective cues as a basis for nestmate recognition in polygynous leptothoracine ants. Proceedings of the National Academy of Sciences of the USA 85: 4572-4575.

Taylor, P. D. 1988. An inclusive-fitness model for dispersal of offspring. Journal of Theoretical Biology 140:363-378.

- 1992. Altruism in viscous populations: an inclusive fitness model. Evolutionary Ecology 6:352-356.

Taylor, P. D., and S. A. Frank. 1996. How to make a kin selection model. Journal of Theoretical Biology 180: 27-37.

Tsuji, K. 1990. Kin recognition in Pristomyrmex pungens (Hymenoptera: Formicidae): asymmetrical changes in acceptance and rejection due to odour transfer. Animal Behaviour 40:306-312.

Tsutsui, N. D., A. V. Suarez, D. A. Holway, and T. J. Case. 2000. Reduced genetic variation and the success of an invasive species. Proceedings of the National Academy of Sciences of the USA 97:5948-5953.

Vienne, C., V. Soroker, and A. Hefetz. 1995. Congruency of hydrocarbon patterns in heterospecific groups of ants: transfer and/or biosynthesis? Insectes Sociaux 42: 267-277.

Wang, J. 1997. Effective size and F-statistics of subdivided populations. II. Dioecious species. Genetics 146: 1465-1474.

Wilson, D. S., G. B. Pollock, and L. A. Dugatkin. 1992. Can altruism evolve in purely viscous populations? Evolutionary Ecology 6:352-356.

Wolfram, S. 1991. Mathematica: a system for doing mathematics by computer. $2 \mathrm{~d}$ ed. Addison-Wesley, Redwood City, Calif. 